\title{
Potential of gene therapy as a treatment for heart failure
}

\author{
Roger J. Hajjar \\ Cardiovascular Research Center, Mount Sinai School of Medicine, New York, New York, USA.
}

\begin{abstract}
Advances in understanding the molecular basis of myocardial dysfunction, together with the evolution of increasingly efficient gene transfer technology, make gene-based therapy a promising treatment option for heart conditions. Cardiovascular gene therapy has benefitted from recent advancements in vector technology, design, and delivery modalities. There is a critical need to explore new therapeutic approaches in heart failure, and gene therapy has emerged as a viable alternative. Advances in understanding of the molecular basis of myocardial dysfunction, together with the development of increasingly efficient gene transfer technology, has placed heart failure within reach of gene-based therapy. The recent successful and safe completion of a phase 2 trial targeting the cardiac sarcoplasmic/endoplasmic reticulum $\mathrm{Ca}^{2+}$ ATPase pump (SERCA2a) has the potential to open a new era for gene therapy for heart failure.
\end{abstract}

\section{Introduction}

Cardiovascular disease is the leading cause of death in the United States (1). Despite significant advancements in promoting cardiovascular health with therapeutic agents and devices as well as improved understanding of the molecular pathways underlying cardiovascular disease, nearly 20 million Americans are diagnosed with coronary heart disease and congestive heart failure (1).

While progress in conventional treatment modalities is making steady and incremental gains to reduce cardiovascular disease burden, an urgent need to explore new therapeutic approaches remains. Gene therapy, which was initially envisioned as a treatment strategy for inherited monogenic disorders, has broad therapeutic potential, including treatment of acquired polygenic diseases such as peripheral vascular disease, ischemic heart disease, arrhythmias, and congestive heart failure (2). Improved understanding of the molecular basis of these conditions, together with the evolution of increasingly safe and efficient gene transfer technologies, has placed cardiovascular disease within reach of gene-based therapies (2).

This Review focuses on gene-modifying strategies that target diseases of the cardiovascular system. The efficacy of various gene and vector delivery systems is addressed with results from human clinical trials. While there are many promising strategies to modify cardiovascular disease, only molecular targets that have progressed to preclinical and human clinical trials are systematically reviewed here. The cardiologist will benefit from an improved understanding the techniques and rationale behind cardiovascular gene therapy as this therapeutic strategy enters the clinical realm.

\section{Gene therapy vectors}

The development of cardiovascular gene transfer intervention necessitates addressing several factors to ensure high efficiency while minimizing toxicity (2). These include understanding target cell and transgene biology and the temporal and spatial patterns of the specific cardiovascular pathophysiological process. In addition, the choices of gene and vector delivery systems also

Conflict of interest: R.J. Hajjar is a scientific founder of Celladon Corp., which plans to commercialize AAV1.SERCA2a for the treatment of heart failure.

Citation for this article: J Clin Invest. 2013;123(1):53-61. doi:10.1172/JCI62837. critically determine clinical outcomes. Answering these questions will dictate the proportion of target cells within the myocardium that need to be successfully gene modified in order to elicit cardioprotection. Restoring myocardial contractility in the context of heart failure requires successful gene transfer to a vast majority of cardiomyocytes in the ventricular myocardium in order to enable a significant effect on ventricular function. Furthermore, the required temporal pattern of transgene expression will determine the choice of gene transfer system that can be employed for efficient and positive results. Gene delivery systems can be classified into two categories, nonviral systems and recombinant viral systems, each of which have unique profiles in gene transfer expression and their own advantages and disadvantages (Figure 1). Nonviral vectors include naked plasmid DNA, liposomal DNA complexes, polymer-carried DNA, and oligonucleotides. Plasmids are double-stranded circular DNA-containing transgenes encoding proteins of interest, and also have enhancer and promoter sequences (2).

Viral vectors from the family Retroviridae include retrovirus and lentivirus $(3,4)$. Retroviruses contain single-stranded, positivesense RNAs that utilize a virally encoded reverse transcriptase to generate double-stranded DNA. In order to enable viral DNA integration into the host cell genome, the host cell nuclear membrane must be broken down, as occurs during cell division; retroviruses are therefore limited to infecting dividing cells and cannot efficiently transduce most cardiomyocytes. Lentiviral vectors, also from the family Retroviridae, are single-stranded RNA viruses utilizing reverse transcriptase and genome integration for the longterm expression of transgenes (4). However, lentiviral vectors are capable of transducing mitotically quiescent cells, allowing for efficient transduction of cardiomyocytes. The random integration afforded by the lentivirus continues to be a concern.

An adenovirus is a non-enveloped, non-integrating virus containing double-stranded DNA with two main transcriptional regions, early and late phase. The early phase encodes E1, E2, $\mathrm{E} 3$, and E4 viral proteins, which are necessary for activating the $\mathrm{S}$ phase of the cell cycle, DNA polymerase, and splicing proteins. The E1-E4 proteins elicit a significant innate immune response, which is the major therapeutic challenge in using adenoviruses in human applications. Third-generation "gutless" adenoviral 


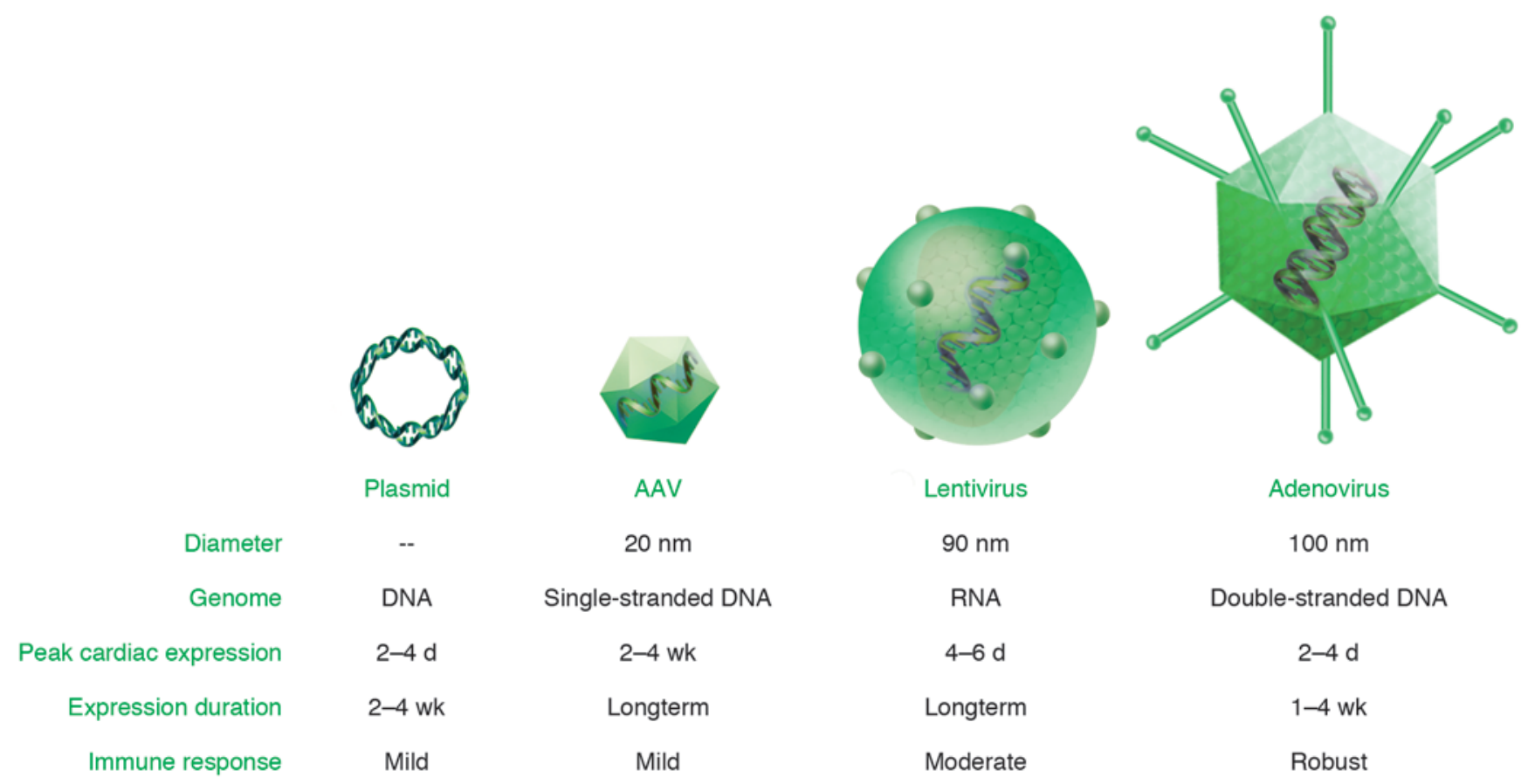

Figure 1

The various vectors commonly used in cardiovascular applications, and their specific characteristics.

vectors with deleted E1-E4 proteins have a lower immunogenicity profile and partially overcome this obstacle (5). In the heart, adenoviral transgene expression is robust but transient (6). Transgene expression levels peaks within two to three days and return to undetectable levels by two weeks. This imposes therapeutic challenges for chronic pathological processes such as congestive heart failure but may be appropriate for short-term, pro-angiogenic responses.

Adeno-associated viruses (AAVs) are members of the family Parvoviridae and are non-enveloped, single-stranded DNA viruses (7). AAVs are relatively small $(20 \mathrm{~nm})$ and therefore are limited in their genome capacity of $4.7 \mathrm{~kb}$. There are 13 reported serotypes of AAV with varying degrees of tissue tropism, depending on capsid protein structure (7). AAV1, $-6,-8$, and -9 have been identified as being the most cardiotropic, but significant transduction persists in nontarget tissues such as liver, skeletal muscle, and lung. Neutralizing antibodies to various AAV serotypes are present in approximately $20 \%-80 \%$ of the population, therefore severely limiting the potential therapeutic use of AAVs, and are a major exclusion criteria in many AAV-based clinical trials $(8,9)$.

The various vector systems all have different expression kinetics and tissue tropisms that must be taken into account when designing human gene therapy trials. Nonspecific expression and offtarget effects are an obstacle for any therapeutic modality.

\section{Gene delivery}

The selection of the appropriate vector delivery method is critical for proper implementation of the therapeutic strategy and for efficient transgene expression in the myocardium $(10,11)$. Vectors have unique profiles of bioavailability, transgene expression kinetics, and tissue tropisms, and it is therefore vital to choose a vector delivery method that complements the vector as well as the disease process. Importantly, the invasiveness of the vector delivery method and patient safety require critical assessment prior to initiating gene therapy trials for cardiovascular disease $(10,11)$.

\section{Coronary artery and venous infusion}

Percutaneous coronary artery catheterization is a minimally invasive and well-established procedure that allows homogeneous gene delivery to each territory of the heart. This approach is relatively safe, making it especially attractive for patients with end-stage heart failure. However, gene delivery can be impeded in patients with severe coronary artery disease. More importantly, some variability regarding the efficiency of antegrade coronary gene transfer exists and is partly related to the relatively fast transit of the vector through the vasculature (ref. 12 and Figure 2).

Enhanced vector residence time in the coronary circulation is achievable with coronary venous blockade. Antegrade coronary infusion with a short occlusion of both a coronary artery and a coronary vein enhanced myocardial gene expression $(13,14)$. This method preserved LV function and inhibited ventricular remodeling in a large animal model of heart failure (14). However, even a short ischemia period carries risk in patients with advanced heart failure.

To maximize the duration of vector exposure to the endothelium while minimizing systemic distribution, Kaye et al. developed a cardiac recirculation approach (15). They engineered an extracorporeal device that drains blood from the coronary sinus using an occlusion catheter and returns the oxygenated coronary venous blood to the left main coronary artery via a peristaltic pump (V-Focus; Osprey Medical Inc.) $(16,17)$. In an ovine model of tachycardia-induced heart failure, the closed-loop recirculation method was more efficient in the transduction of cardiomyocytes than antegrade coronary infusion, which also translated into a 
A

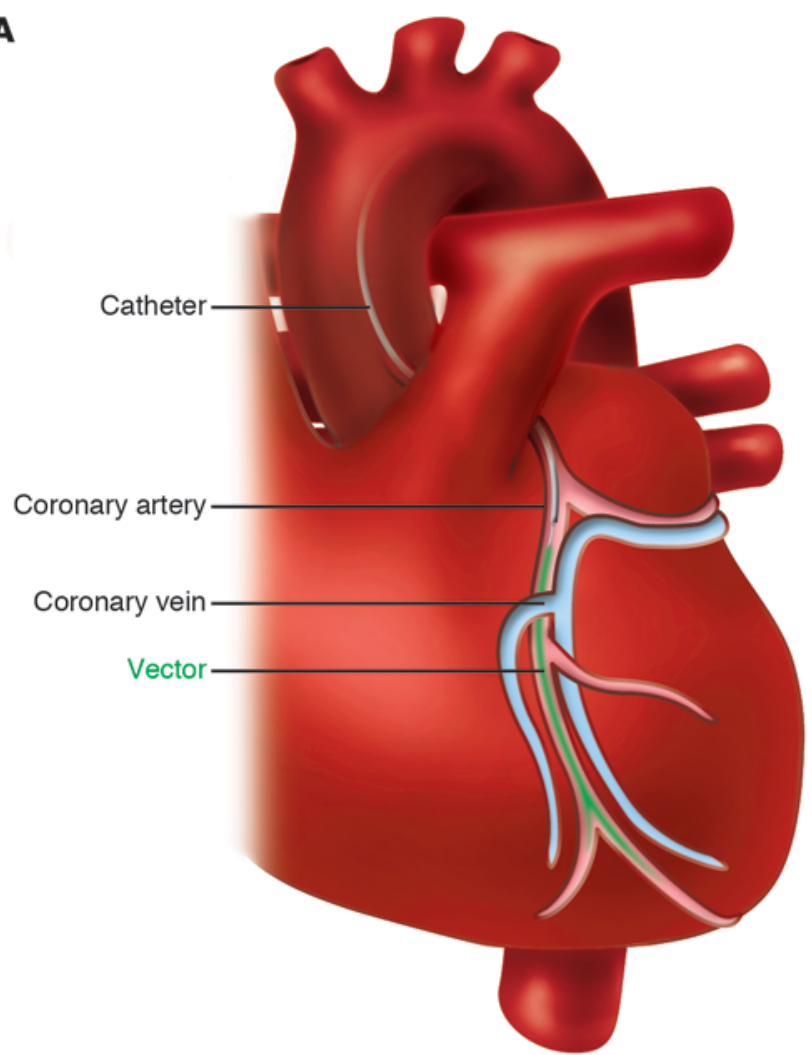

B

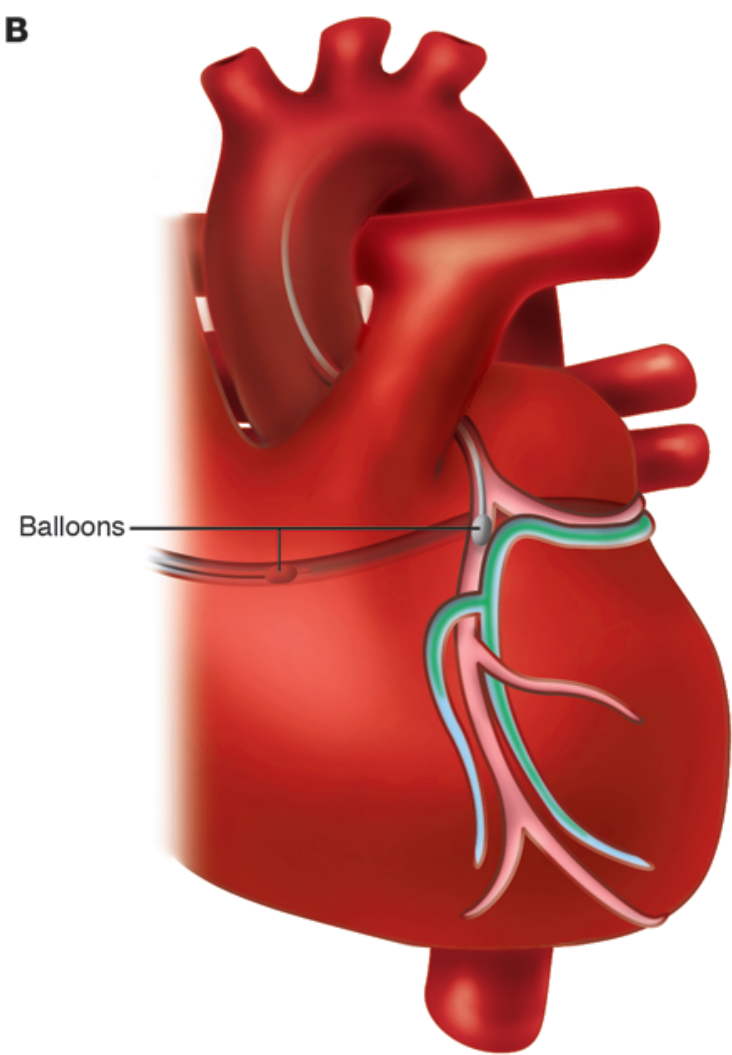

Figure 2

Infusion-based techniques for gene delivery to the heart. (A) Coronary artery infusion. The vector is injected through a catheter, without interruption of the coronary flow, using a slow infusion. (B) Retrograde coronary venous infusion with simultaneous blocking of a coronary artery and a coronary vein. The vector is injected into a coronary vein and resides in the coronary circulation until both balloons are deflated.

greater improvement of LV function $(15,16)$. In addition, this method allows the selective administration of endothelial permeabilizing agents (18) without systemic side effects.

Most approaches to improve the efficiency of coronary artery infusion are based on increasing the exposure time of the vector to the endothelium. However, transduction efficacy correlates with coronary flow as well as exposure time and vector concentration (19). Based on the former, antegrade coronary artery infusion supported by an increased coronary flow, for example an intra-aortic balloon pump, might further enhance cardiac gene transfer (20). Antegrade coronary artery infusion might be the safest percutaneous gene delivery method, but at the cost of efficiency relative to other techniques. However, to date, all optimization strategies implicate potential damage to the myocardium, limiting their clinical application.

The coronary venous system provides an alternate route for the percutaneous delivery of therapeutic agents to the myocardium. In a clinical setting, this approach is attractive for patients with impaired coronary artery circulation and limited potential for revascularization. Access to the myocardium can be achieved with this approach regardless of atherosclerosis severity or coronary artery obstruction. Numerous studies showed that retrograde coronary venous infusion is an alternate myocardial delivery method for cardioprotective drugs $(21,22)$. Shortly thereafter, retrograde venous infusion was also explored as a method for gene transfer. The rationale for a high transduction efficiency is based on controlling the exposure time of the vector to the endothelium and on increasing the pressure gradient of capillary filtration $(23,24)$.

Studies in large animal models demonstrated that an efficient and homogeneous myocardial transduction can be achieved by retroinfusion into the coronary venous system (24-26). Boekstegers et al. showed that gene expression after pressure-regulated retrograde venous infusion was significantly higher than after antegrade coronary delivery if the retroinfusion was accompanied by simultaneous induced ischemia (ref. 24 and Figure 2B). Compared with percutaneous or surgical direct myocardial injection, retrograde venous infusion also achieved more homogeneous and efficient reporter gene expression (25).

Similar to antegrade coronary artery infusion, a closed loop recirculation retrograde venous infusion approach is also feasible (27). Recently, White et al. demonstrated an extremely high transduction efficiency in the majority of cardiomyocytes in sheep while minimizing collateral organ exposure using a retrograde recirculation method during cardiopulmonary bypass surgery (28). Retrograde coronary infusion has been shown to be safe, when performed by a trained and experienced surgeon. Trauma to the coronary veins, a potential complication, could be minimized with modifications and improvements in catheter design. However, the procedure is not completely without risks. Highly elevated coronary venous pressure can result in myocardial edema or hemorrhage. Careful pressure monitoring should always 

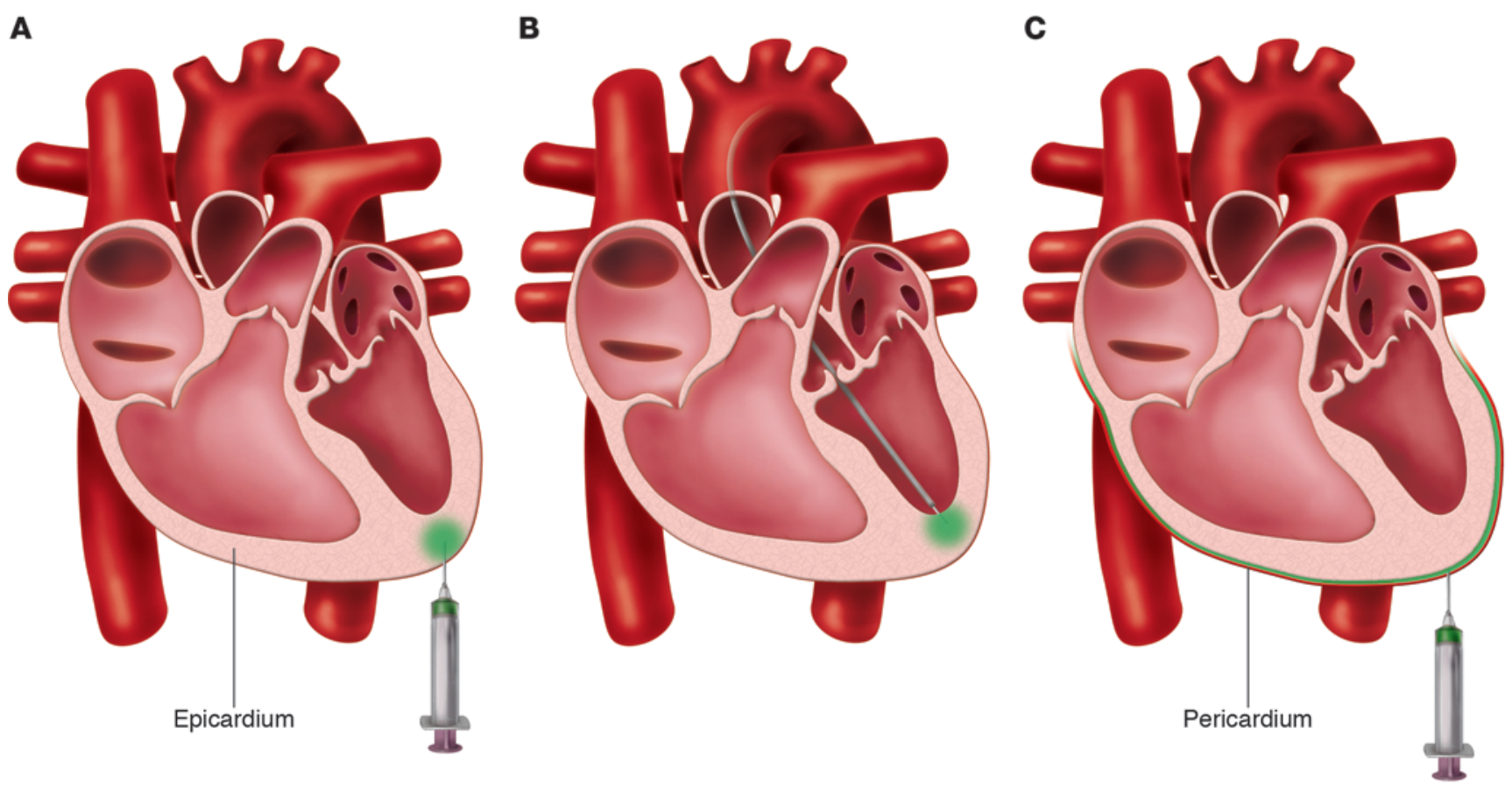

Figure 3

Direct myocardial injection and pericardial injection methods of gene delivery. (A) Percutaneous myocardial injection. The vector is injected with an injection catheter via an endocardial approach. (B) Surgical myocardial injection. The vector is injected via an epicardial approach. (C) Percutaneous pericardial injection; the vector is injected in the pericardial space.

be used to avoid this complication. Although, with the growing demand for cardiac resynchronization therapy, cannulation of the coronary veins has become a routine procedure, the monitoring of pressure regulation adds to the complexity of this procedure. More importantly, ischemia during the infusion might not be well tolerated in patients with advanced heart failure. While relatively complex, percutaneous retrograde coronary infusion is an effective and minimally invasive procedure for gene delivery to the myocardium. This approach requires some expertise and meticulous pressure monitoring to be performed safely.

\section{Direct intramyocardial injection}

Intramyocardial injection is one of the most widely used gene transfer methods in both small animal studies as well as clinical trials focused on cardiac angiogenesis. The vectors are injected either epicardially (Figure 3A) or endocardially (Figure 3B) into the target area with a small-gauge needle. The primary advantage of this method is that vector delivery bypasses the endothelial barrier. This results in a high local concentration at the injection site. In addition, by avoiding exposure to the blood, deactivation of the vectors by circulating DNases or neutralizing antibodies can be prevented. Furthermore, there is minimal exposure of the vector to off-target organs, although local administration cannot completely avoid some systemic vector distribution $(29,30)$. Low volumes at high vector concentrations may increase the vector retention in the myocardium (29).

The simplest approach, however invasive, is the injection during the thoracotomy (31). Surgical delivery offers direct visual confirmation, which allows precise control of the injection site, including an infarct border.
The endocardial approach requires a catheter with a retractable injection needle and imaging guidance modality for determining the injection site (30). This includes electrical mapping systems (32), fluoroscopy (33), echocardiography (34), and magnetic resonance imaging (35). Currently, the NOGA electromechanical mapping system is the most commonly used guiding system that is also used in clinical angiogenesis trials.

Intramyocardial injection is attractive for local gene delivery. However, the application of this method for heart failure, which is associated with global impairments in cardiomyocytes, might be limited by a circumscribed target area and inhomogeneous expression profiles $(25,31)$.

Intrapericardial delivery is performed surgically in rodents (36), whereas a percutaneous approach is also possible in larger animals (Figure 3C). The percutaneous pericardial puncture has been proven to be feasible and safe when guided by imaging techniques such as fluoroscopy and intravascular ultrasound. The percutaneous access to the pericardial space can be minimally invasive via a substernal/xhiphoidal puncture. A transatrial approach has also been described (37), but this technique can result in more fibrosis and scar tissue compared with the substernal approach.

The pericardial space faces most of the cardiac wall, except the septum. In heart failure, in which widespread cardiac gene transfer with little systemic distribution is desired, this large-scale interface combined with the concept of a closed compartment can be a major advantage. These features potentially enable prolonged vector persistence, a slow release over time, a high vector concentration, and minimal leakage to nontarget organs. However, tightly joined pericardial cells restrict transfection to superficial 


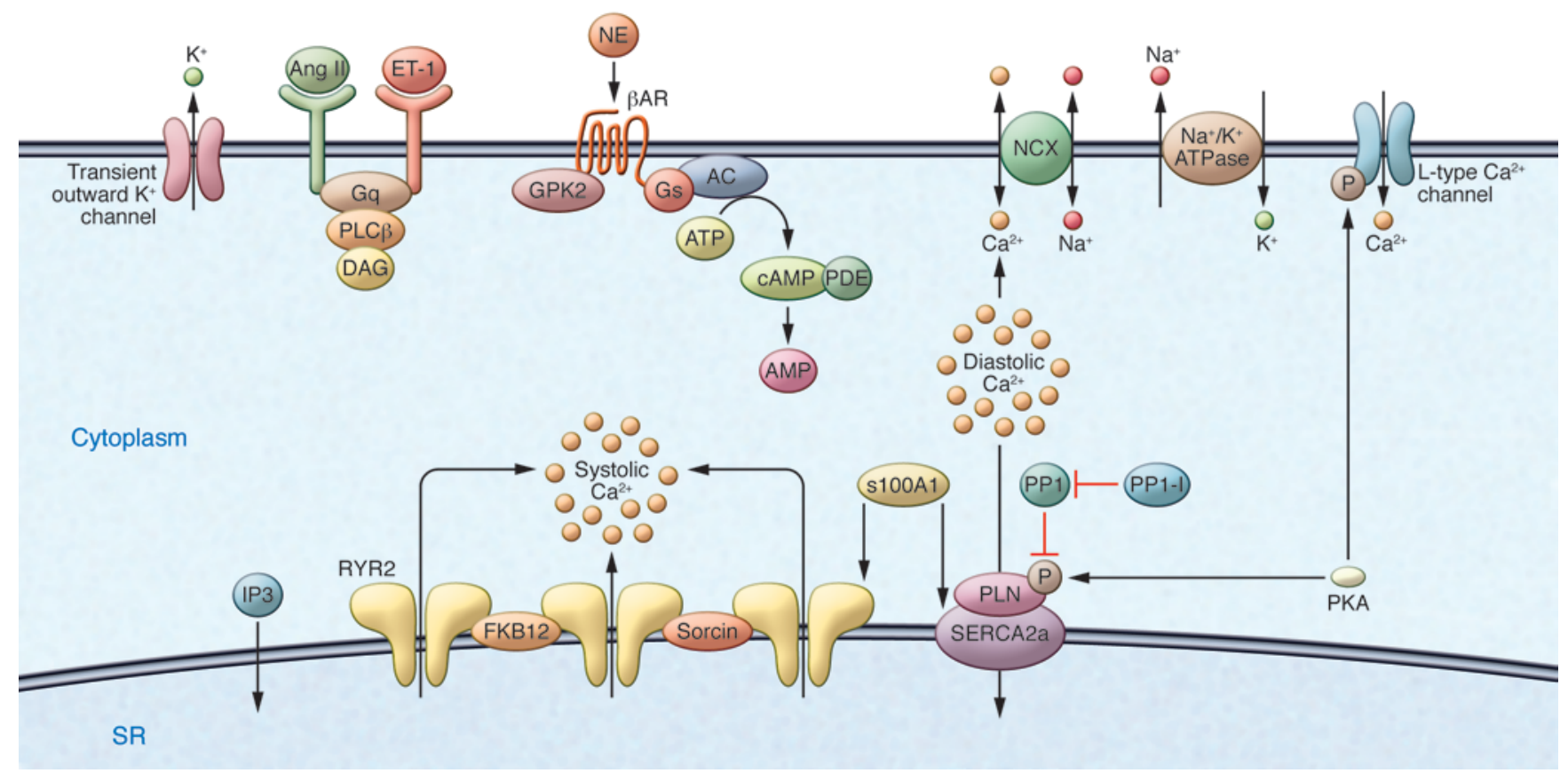

Figure 4

Excitation-contraction coupling in cardiomyocytes, with multiple targets for genetic modulation. Strategies for improving calcium cycling within the cardiac cell have focused on pharmacologically inhibiting the $\mathrm{Na} / \mathrm{K}$ ATPase, which results in increased intracellular $\mathrm{Ca}^{2+}$; more recently, strategies have focused on stabilizing the RYR, resulting in decreased sarcoplasmic reticulum $\mathrm{Ca}^{2+}$ leak. Gene editing techniques have focused on enhancing the activity of SERCA2a by either increasing the level of SERCA2a or altering the expression of its partners.

myocardial layers $(36,38,39)$. This limitation can be partly overcome by the co-administration of various pharmacological agents. Proteolytic enzymes and polyethyleneimine have been shown to increase the penetration depth of the vectors and to allow progressive release, often at the cost of cardiac toxicity $(36,40)$. Although vectors are injected into a closed space, some studies reported extracardiac gene expression, probably due to the rapid turnover of the pericardium fluid through the lymphatic absorption (41). A platform that allows slow vector release together with a permeabilizing, nontoxic agent may increase the potential of pericardial injection for gene delivery (42).

The combination of echocardiography contrast microbubbles with a viral vector delivery system has been shown to provide efficient gene transduction to the myocardium $(43,44)$. However, there is some concern that microbubble destruction by echocardiography can cause endothelial damage.

\section{Molecular targets}

Over the last 20 years, there has been significant evolution in our understanding of the pathophysiology of heart failure in its molecular and cellular dimensions, thus leading to a broader scope of interventions available for gene therapy. For targets to be validated, it is important that they rescue function in animal models in which heart failure has been already established, that the rescue is not associated with arrhythmogenesis, and that a gene-dose effect is established (i.e., with increasing gene expression, a concomitant improvement in function is observed). Excitation-contraction coupling is dysregulated at multiple levels in the development of heart failure. For this reason, the various channels, transporters, and critical proteins have been targeted pharmacologically and by genetic editing to restore contractile function. In Figure 4, the various targets in excitation-contraction coupling are presented.

Targeting the $\beta$-adrenergic system. The $\beta$-adrenergic signaling is adversely affected by multiple changes that lead to $\beta$-adrenergic receptor $(\beta-A R)$ downregulation and de-sensitization. Upregulation of the critical protein $G$ protein-coupled receptor kinase 2 (GRK2) seems to precipitate the abnormalities in $\beta$-AR signaling abnormalities. Several gene-based experiments have tested the hypothesis that genetic manipulation of the myocardial $\beta$-AR system can enhance cardiac function.

Overexpression of $\beta$-AR was initially tested as a simple way to overcome $\beta$-AR downregulation. Transgenic mice overexpressing the human $\beta_{1}$-ARs suffered from severe cardiomyopathy (45). In contrast, mice with cardiac overexpression of $\beta_{2}$-AR demonstrated increased basal myocardial adenylyl cyclase activity with increased LV function (46). Both direct and intracoronary myocardial delivery of adenovirus containing the human $\beta_{2}$-AR transgene resulted in enhanced cardiac performance in rodents and mammalian models $(47,48)$.

The interaction between activated $\beta$-ARs and $G$ proteins is regulated by kinases that modulate the receptor activity by phosphorylation of its carboxyl terminus. Agonist-dependent desensitization is mediated by a family of GRKs that phosphorylate the agonist-occupied receptors, resulting in functional uncoupling. GRK2 binds to the $G \beta \gamma$ subunit of activated $G$ proteins, phosphorylating $\beta$-ARs that subsequently attach to the inhibitory protein $\beta$-arrestin. GRK2, the most abundantly expressed GRK in the heart, has been implicated in the pathogenesis of dysfunctional cardiac $\beta$-AR signaling, accounting for a deleterious activity in 


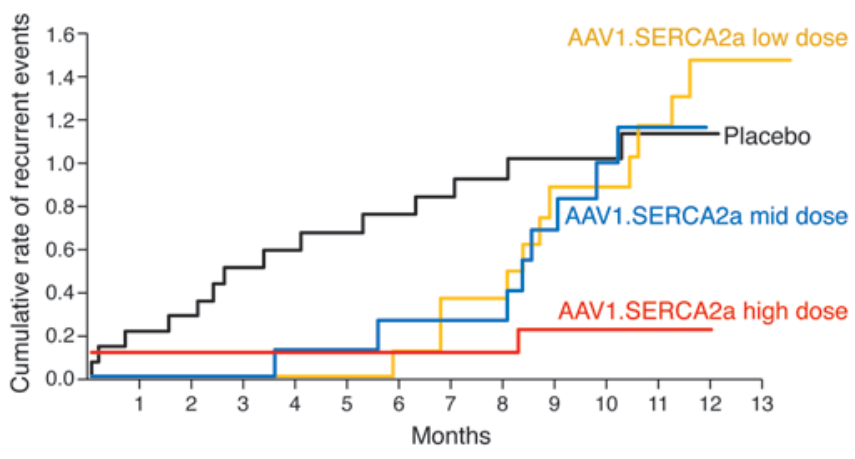

Figure 5

Cumulative clinical event rates, adjusted for the competing risk of terminal events, in patients followed for 12 months in the phase 2 segment of the CUPID trial. Modified with permission from Circulation (88).

the failing heart (49). In mice, selective GRK2 ablation following myocardial infarction-induced heart failure resulted in increased survival, halted ventricular remodeling, and enhanced cardiac contractile performance (50). $\beta A R K c t$, a peptide capable of inhibiting GRK2 mediated $\beta$-AR desensitization, has been evaluated in vivo in animals. Using intracoronary adenovirus-mediated $\beta$ ARKct transgene delivery to rabbits three weeks after induced myocardial infarction demonstrated a marked reversal of ventricular dysfunction (51). More recent studies have focused on overexpressing $\beta A R K c t$ in large animal models (52).

Although detrimental outcomes were demonstrated with multiple elements of the $\beta$-adrenergic system used to improve the expression of cAMP, activation of adenylate cyclase 6 (ADCY6) seems to have a unique and favorable profile. Overexpression of ADCY6 in transgenic mice resulted in improved cardiac function in response to adrenergic stimulation along with increased cAMP production in isolated cardiomyocytes. Importantly, ADCY6 had a neutral effect on basal heart function and was not associated with any structural heart abnormalities (53). In a pacing model of heart failure in pigs, intracoronary delivery of adenovirus encoding ADCY6 resulted in improved LV function and remodeling, associated with increased cAMP-generating capacity (54). The favorable effects of ADCY 6 in preclinical studies are encouraging, and this approach is currently under investigation for initiation of clinical trials in patients with heart failure (55).

Targeting $\mathrm{Ca}^{2+}$ cycling proteins. Heart failure is characterized by multiple defects in $\mathrm{Ca}^{2}$-handling proteins involved in excitationcontraction coupling (Figure 4). Reversal of those defects by gene therapy techniques has shown very promising results.

More than 20 years ago, Gwathmey et al. first reported that calcium cycling is abnormal in human heart failure (56); this is partially due to decreased sarcoplasmic/endoplasmic reticulum $\mathrm{Ca}^{2+}$ ATPase 2a (SERCA2a) activity regardless of the etiology of the heart failure (57-60). Improvement in cardiac contractility after gene transfer of SERCA2 $a$ has been demonstrated in a large number of experimental models of heart failure $(61,62)$. More importantly, long-term overexpression of SERCA2a by intracoronary delivery of AAVs carrying SERCA2 $a$ was associated with preserved systolic function and improved ventricular remodeling in a swine model of volume-overload heart failure (63). Beyond their effects on enhancing contractility, SERCA2a gene transfer has been shown to (a) restore the normal state of cardiac energetics $(64,65)$ in terms of energy supply and utilization, (b) decrease ventricular arrhythmias (66-69), and (c) enhance coronary flow through activation of eNOS in endothelial cells (70).

Another approach to improve $\mathrm{Ca}^{2+}$ handling involves inhibition of phospholamban (PLN). The lowering of PLN levels in human cardiomyocytes resulted in improved contraction and relaxation velocities similar to the benefit seen with gene transfer of SERCA2a (71). Silencing of PLN expression in a sheep model of heart failure resulted in improved SERCA activity along with improved systolic and diastolic LV function (15). In addition to the above conventional gene therapy strategies, RNAi therapy was used for the first time in a model of cardiac disease, specifically in rats with heart failure, in an attempt to suppress PLN expression. An AAV9 RNAi vector generated stable cardiac production of a regulatory RNA sequence, which in turn suppressed PLN expression. SERCA2a protein levels were subsequently increased, accompanied by the restoration of systolic and diastolic cardiac function (72).

Heart failure is also associated with elevated protein phosphatase 1 (PP1) activity in humans, which results in dephosphorylation of PLN. Overexpression of PP1 or ablation of inhibitor of PP1 (I-1) in murine hearts has been associated with decreased $\beta$-AR-mediated contractile responses, depressed cardiac function, and premature death consistent with heart failure (73-75). Expression of a constitutively active I- 1 in transgenic mice led to PP1 inhibition with increased phosphorylation of PLN and improved cardiac contractility. A recent study on transgenic mice expressing active I-1 confirmed the relationship between phosphorylation of PLN and SERCA2a activity. I-1 expression ameliorated ischemia/reperfusion-induced injury by reducing the infarct size and improving contractile recovery in addition to decreasing biomarkers of apoptosis and ER stress response (73-75).

$\mathrm{S} 100$ is part of a family of $\mathrm{Ca}^{2+}$-modulated proteins implicated in intracellular regulatory activities. S100A1 is the most abundant $\mathrm{S} 100$ protein isoform in the heart. It promotes cardiac contractile and relaxation function by enhancing the activity of both ryanodine receptors (RYRs) and SERCA2a (76). In a rat model of heart failure, AAV6-mediated long-term expression of S100A1 resulted in a sustained in vivo reversal of $\mathrm{LV}$ dysfunction and remodeling $(77,78)$. More recently AAV9 gene transfer of S100A1 in a preclinical model of ischemic cardiomyopathy induced dramatic improvements in contractile function, reinforcing the rationale that a clinical trial of S100A1 gene therapy for human heart failure should be forthcoming.

Recently Kho et al. reported that the levels and activity of SERCA2a in cardiomyocytes are modulated in parallel with the levels of small ubiquitin-related modifier 1 (SUMO1) (79). SUMOs are a family of peptides that broadly alter the function of other proteins in cells through a post-translational modification known as sumoylation. Kho et al. found that sumoylation enhances the stability of SERCA2a in the cell as well as increases its activity. SUMO1 levels were reduced in murine and pig models of heart failure and in failing human ventricles. Increasing SUMO1 levels by AAV9 gene transfer led to a restoration of SERCA2a levels, improved hemodynamic performance, and reduced mortality among the animals with heart failure.

Lastly, the SDF1/CXCR4 complex has emerged as a therapeutic target in ischemic heart failure (80) due to the ability of the SDF1/CXCR4 system to promote the homing of stem cells to infracted myocardium. A clinical trial is underway to investigate the therapeutic benefit of SDF1 overexpression in ischemic 
cardiomyopathy (81). In parallel, existing literature highlights the direct effects of CXCR4 on the myocardium and the cardiomyocyte. SDF1 was shown to decrease myocardial contractility ex vivo and on cardiomyocytes (82). One recent report has shown increased ischemia-reperfusion injury in rat hearts overexpressing CXCR4 (83), while another report investigated the modulation of $\beta$-AR signaling by SDF1 and CXCR4 (84), raising interrogations over the potential complex interaction between these chemokines and the cardiovascular system.

\section{Clinical trials}

After years of consecutive failures, gene therapy trials for specific monogenic diseases have shown some successes, most notably for inherited blindness in a pediatric patient with Leber congenital amaurocis (85) and in hemophilia.

The first clinical trial of gene therapy in patients with heart failure was launched in the United States in 2007 (86, 87). Calcium Up-regulation by Percutaneous Administration of Gene Therapy in Cardiac Disease (CUPID) was a two-part, Phase $1 / 2$ multicenter trial designed to evaluate the safety and the biological effects of gene transfer of the SERCA2a cDNA by delivering a recombinant AAV1 (AAV1.SERCA2a) in patients with advanced heart failure. In part 1, participants in this trial were administered a single intracoronary infusion of AAV1.SERCA2a in an open-label approach $(86,87)$. Four cohorts of three patients each received a single escalating dose of AAV1.SERCA2a. Twelve-month follow-up of these patients showed an acceptable safety profile $(86,87)$. Improvement was detected in several patients, as reflected by symptomatic (five patients), functional (four patients), biomarker (two patients), and LV function/remodeling (six patients) parameters. Although part 1 was a phase 1 study involving a small number of patients, the results suggested that AAV1.SERCA2a treatment confers quantitative biological benefit.

In part 2 of the trial, 39 patients with advanced heart failure were randomized to receive intracoronary AAV1-mediated SERCA2 a gene delivery in one of three doses (low dose: $6 \times 10^{11}$ DRP, middle dose: $3 \times 10^{12} \mathrm{DRP}$, and high dose: $\left.1 \times 10^{13} \mathrm{DRP}\right)$ or placebo (88). Patients' symptoms (New York Heart Association (NYHA) classification and Minnesota Living With Heart Failure Questionnaire [MLWHFQ] score), functional status (six-minute walk test [6MWT] and $\mathrm{VO}_{2} \mathrm{max}$ ), NT-proBNP levels, and echocardiographic measures were evaluated over six months. Treatment success was determined by evaluating clinical outcomes and concordant trends in the above endpoints for group- and patientbased comparisons. The AAV1.SERCA2a high-dose group met the pre-specified criteria for success at the group and individual patient levels. Compared with patients treated with placebo, those treated with AAV1.SERCA2a demonstrated improvement or stabilization in NYHA class, MLWHFQ, $6 \mathrm{MWT}, \mathrm{VO}_{2} \mathrm{max}$, NT-proBNP levels, and LV end-systolic volumes at 12 months. Over a one-year period, cumulative recurrent cardiovascular events (death, heart failure admission, LV assist device insertion, cardiac transplantation, and myocardial infarction) increased in the placebo group, as shown in Figure 5. The low- and middledose AAV1.SERCA2a groups had diminished recurrent cardiovascular events for the first six months, and from months 6 to 12 had events that were similar to placebo. The high-dose AAV1. SERCA2a group, however, continued to do significantly better than all groups at 12 months, as shown in Figure 5. No increases in adverse events, disease-related events, laboratory abnormalities, or arrhythmias were observed in AAV1.SERCA2a-treated patients compared with placebo (88).

Two other clinical trials targeting SERCA2a will be enrolling patients soon. The first trial, which is being conducted in the United Kingdom, is in patients with advanced heart failure who received LV assist devices at least one month prior to treatment and who will receive be treated with AAV1.SERCA2a or saline. A phase 2, single-center, double-blind, randomized, placebo-controled, parallel-group study will be conducted at the Institut de Cardiologie Pitié-Salpêtrière in Paris, France, with the primary objective of investigating the impact of AAV1.SERCA2a on cardiac remodeling parameters in patients with severe heart failure.

In another ongoing clinical study that is currently enrolling patients, adenovirus 5-encoding human ADCY6 is delivered through intracoronary injection of Ad5.hAC6 or PBS with dose escalation to patients with congestive heart failure.

An additional trial that is currently recruiting patients will examine the effects of injecting SDF1 directly into the myocardium of patients with ischemic heart disease. In this open-label trial, the safety will be evaluated of an endomyocardial injection of a single escalating dose of SDF1-naked DNA at multiple sites using a percutaneous LV approach, to cohorts of adults with ischemic heart failure.

\section{Conclusion}

As our understanding of the molecular mechanisms associated with heart failure has improved and vectors with cardiotropic properties are being developed, gene therapy can now be considered a viable adjunctive treatment to mechanical and pharmacological therapies for heart failure. In the coming years, additional targets will emerge that are amenable to genetic manipulations, as well as more advanced vector systems that will undoubtedly lead to safer and more effective clinical trials in gene therapy for heart failure.

\section{Acknowledgments}

This work is supported by NIH grants R01 HL093183, HL088434, and P20HL100396; Program of Excellence in Nanotechnology contract HHSN26820100045c; and P50HL 112324.

Address correspondence to: Roger J. Hajjar, Cardiovascular Research Center, Mount Sinai School of Medicine, One Gustave Levy Place, Box 1030, New York, New York 10029, USA. Phone: 212.241.4082; Fax: 212.241.4080; E-mail: roger.hajjar@mssm.edu.
1. Roger VL, et al. Heart disease and stroke statistics-2012 update: a report from the American Heart Association. Circulation. 2012;125(1):e2-e220.

2. Tilemann L, Ishikawa K, Weber T, Hajjar RJ. Gene therapy for heart failure. Circ Res. 2012; 110(5):777-793.

3. Trono D. Lentiviral vectors: turning a deadly foe into a therapeutic agent. Gene Ther. 2000;7(1):20-23.

4. Bonci D, et al. 'Advanced' generation lentivi- ruses as efficient vectors for cardiomyocyte gene transduction in vitro and in vivo. Gene Ther. 2003; 10(8):630-636.

5. Vetrini F, Ng P. Gene therapy with helper-dependent adenoviral vectors: current advances and future perspectives. Viruses. 2010;2(9):1886-1917.

6. Giordano FJ, et al. Intracoronary gene transfer of fibroblast growth factor-5 increases blood flow and contractile function in an ischemic region of the heart. Nat Med. 1996;2(5):534-539.

7. Asokan A, Schaffer DV, Samulski RJ. The AAV vector toolkit: poised at the clinical crossroads. $\mathrm{Mol}$ Ther. 2012;20(4):699-708.

8. Li C, et al. Neutralizing antibodies against adenoassociated virus examined prospectively in pediatric patients with hemophilia. Gene Ther. 2012; 19(3):288-294.

9. Calcedo R, Vandenberghe LH, Gao G, Lin J, 
Wilson JM. Worldwide epidemiology of neutralizing antibodies to adeno-associated viruses. J Infect Dis. 2009;199(3):381-390.

10. Ladage D, Ishikawa K, Tilemann L, Muller-Ehmsen J, Kawase Y. Percutaneous methods of vector delivery in preclinical models. Gene Ther. 2012 19(6):637-641.

11. Ishikawa $\mathrm{K}$, et al. Cardiac gene therapy in large animals: bridge from bench to bedside. Gene Ther. 2012 19(6):670-677.

12. Parsa CJ, et al. Catheter-mediated subselective intracoronary gene delivery to the rabbit heart: introduction of a novel method. J Gene Med. 2005; 7(5):595-603.

13. Hayase $M$, et al. Catheter-based antegrade intracoronary viral gene delivery with coronary venous blockade. Am J Physiol Heart Circ Physiol. 2005; 288(6):H2995-H3000.

14. Beeri R, et al. Gene delivery of sarcoplasmic reticulum calcium ATPase inhibits ventricular remodeling in ischemic mitral regurgitation. Circ Heart Fail. 2010;3(5):627-634

15. Kaye DM, et al. Percutaneous cardiac recirculationmediated gene transfer of an inhibitory phospholamban peptide reverses advanced heart failure in large animals. J Am Coll Cardiol. 2007;50(3):253-260.

16. Byrne MJ, Power JM, Preovolos A, Mariani JA, Hajjar RJ, Kaye DM. Recirculating cardiac delivery of AAV2/1SERCA2a improves myocardial function in an experimental model of heart failure in large animals. Gene Ther. 2008;15(23):1550-1557.

17. Mariani JA, Smolic A, Preovolos A, Byrne MJ, Power JM, Kaye DM. Augmentation of left ventricular mechanics by recirculation-mediated AAV2/ 1-SERCA2a gene delivery in experimental heart failure. Eur J Heart Fail. 2011;13(3):247-253.

18. Sasano T, Kikuchi K, McDonald AD, Lai S, Donahue JK. Targeted high-efficiency, homogeneous myocardial gene transfer. J Mol Cell Cardiol. 2007; 42(5):954-961

19. Donahue JK, Kikkawa K, Johns DC, Marban E, Lawrence JH. Ultrarapid, highly efficient viral gene transfer to the heart. Proc Natl Acad Sci US A. 1997;94(9):4664-4668.

20. Ladage D, et al. Increasing the efficacy of myocardial gene therapy with an intra-aortic balloon pump in a large animal model of heart failure. Eur Heart J. 2011;32(abstract supplement):814.

21. Ryden L, et al. Pharmacokinetic analysis of coronary venous retroinfusion: a comparison with anterograde coronary artery drug administration using metoprolol as a tracer. J Am Coll Cardiol. 1991; 18(2):603-612.

22. Karagueuzian HS, et al. Coronary venous retroinfusion of procainamide: a new approach for the management of spontaneous and inducible sustained ventricular tachycardia during myocardial infarction. J Am Coll Cardiol. 1986;7(3):551-563.

23. Su LT, et al. Uniform scale-independent gene transfer to striated muscle after transvenular extravasation of vector. Circulation. 2005;112(12):1780-1788.

24. Boekstegers P, et al. Myocardial gene transfer by selective pressure-regulated retroinfusion of coronary veins. Gene Ther. 2000;7(3):232-240.

25. Raake $P$, et al. Myocardial gene transfer by selective pressure-regulated retroinfusion of coronary veins: comparison with surgical and percutaneous intramyocardial gene delivery. J Am Coll Cardiol. 2004; 44(5):1124-1129.

26. Hou D, et al. Widespread regional myocardial transfection by plasmid encoding Del-1 following retrograde coronary venous delivery. Catheter Cardiovasc Interv. 2003;58(2):207-211.

27. Davidson MJ, et al. Cardiac gene delivery with cardiopulmonary bypass. Circulation. 2001; 104(2):131-133.

28. White JD, et al. Myocardial gene delivery using molecular cardiac surgery with recombinant adeno- associated virus vectors in vivo. Gene Ther. 2011; 18(6):546-552.

29. Grossman PM, Han Z, Palasis M, Barry JJ, Lederman RJ. Incomplete retention after direct myocardial injection. Catheter Cardiovasc Interv. 2002; 55(3):392-397

30. Bish LT, et al. Percutaneous transendocardial delivery of self-complementary adeno-associated virus 6 achieves global cardiac gene transfer in canines. Mol Ther. 2008;16(12):1953-1959.

31. French BA, Mazur W, Geske RS, Bolli R. Direct in vivo gene transfer into porcine myocardium using replication-deficient adenoviral vectors. Circulation. 1994;90(5):2414-2424.

32. Gepstein L, Hayam G, Ben-Haim SA. A novel method for nonfluoroscopic catheter-based electroanatomical mapping of the heart. In vitro and in vivo accuracy results. Circulation. 1997;95(6):1611-1622.

33. Sanborn TA, et al. Percutaneous endocardial transfer and expression of genes to the myocardium utilizing fluoroscopic guidance. Catheter Cardiovasc Interv. 2001;52(2):260-266.

34. Baklanov DV, et al. Live 3D echo guidance of catheter-based endomyocardial injection. Catheter Cardiovasc Interv. 2005;65(3):340-345.

35. Lederman RJ, et al. Catheter-based endomyocardial injection with real-time magnetic resonance imaging. Circulation. 2002;105(11):1282-1284

36 . Fromes Y, et al. Gene delivery to the myocardium by intrapericardial injection. Gene Ther. 1999; 6(4):683-688

37. Waxman S, Moreno R, Rowe KA, Verrier RL. Persistent primary coronary dilation induced by transatrial delivery of nitroglycerin into the pericardial space: a novel approach for local cardiac drug delivery. J Am Coll Cardiol. 1999;33(7):2073-2077.

38. Lamping KG, Rios CD, Chun JA, Ooboshi H, Davidson BL, Heistad DD. Intrapericardial administration of adenovirus for gene transfer. Am J Physiol. 1997;272(1 pt 2):H310-H317.

39. Ladage D, et al. Delivery of gelfoam-enabled cells and vectors into the pericardial space using a percutaneous approach in a porcine model. Gene Ther. 2011; 18(10):979-985

40. Roques C, Salmon A, Fiszman MY, Fattal E, Fromes $Y$. Intrapericardial administration of novel DNA formulations based on thermosensitive Poloxamer 407 gel. Int J Pharm. 2007;331(2):220-223.

41. Boulanger B, Yuan Z, Flessner M, Hay J, Johnston M. Pericardial fluid absorption into lymphatic vessels in sheep. Microvasc Res. 1999;57(2):174-186.

42. Ladage D, et al. Delivery of gelfoam-enabled cells and vectors into the pericardial space using a percutaneous approach in a porcine model. Gene Ther. 2011; 18(10):979-985.

43. Shohet RV, Grayburn PA. Potential bioeffects of ultrasonic destruction of microbubble contrast agents. J Am Coll Cardiol. 2006;47(7):1469-1470.

44. Beeri R, Guerrero JL, Supple G, Sullivan S, Levine RA, Hajjar RJ. New efficient catheter-based system for myocardial gene delivery. Circulation. 2002; 106(14):1756-1759.

45. Engelhardt S, Hein L, Dyachenkow V, Kranias EG, Isenberg G, Lohse MJ. Altered calcium handling is critically involved in the cardiotoxic effects of chronic beta-adrenergic stimulation. Circulation. 2004;109(9):1154-1160.

46. Milano CA, et al. Enhanced myocardial function in transgenic mice overexpressing the beta 2 -adrenergic receptor. Science. 1994;264(5158):582-586.

47. Maurice JP, et al. Enhancement of cardiac function after adenoviral-mediated in vivo intracoronary beta2-adrenergic receptor gene delivery. J Clin Invest. 1999;104(1):21-29.

48. Shah AS, et al. Intracoronary adenovirus-mediated delivery and overexpression of the beta(2)adrenergic receptor in the heart: prospects for molecular ventricular assistance. Circulation. 2000;
101(4):408-414.

49. Hata JA, Williams ML, Koch WJ. Genetic manipulation of myocardial beta-adrenergic receptor activation and desensitization. J Mol Cell Cardiol. 2004; 37(1):11-21.

50. Raake PW, et al. G protein-coupled receptor kinase 2 ablation in cardiac myocytes before or after myocardial infarction prevents heart failure. Circ Res. 2008; 103(4):413-422.

51. Shah AS, et al. In vivo ventricular gene delivery of a beta-adrenergic receptor kinase inhibitor to the failing heart reverses cardiac dysfunction. Circulation. 2001;103(9):1311-1316

52. Katz MG, et al. AAV6-betaARKct gene delivery mediated by molecular cardiac surgery with recirculating delivery (MCARD) in sheep results in robust gene expression and increased adrenergic reserve. J Thorac Cardiovasc Surg. 2012;143(3):720-726

53. Gao MH, et al. Adenylylcyclase increases responsiveness to catecholamine stimulation in transgenic mice. Circulation. 1999;99(12):1618-1622

54. Lai NC, et al. Intracoronary adenovirus encoding adenylyl cyclase VI increases left ventricular function in heart failure. Circulation. 2004;110(3):330-336.

55. Lai NC, et al. Intracoronary delivery of adenovirus encoding adenylyl cyclase VI increases left ventricular function and cAMP-generating capacity. Circulation. 2000;102(19):2396-2401.

56. Gwathmey JK, et al. Abnormal intracellular calcium handling in myocardium from patients with end-stage heart failure. Circ Res. 1987;61(1):70-76.

57. Hasenfuss $\mathrm{G}$, et al. Relation between myocardial function and expression of sarcoplasmic reticulum $\mathrm{Ca}(2+)$-ATPase in failing and nonfailing human myocardium. Circ Res. 1994;75(3):434-442.

58. Meyer M, et al. Alterations of sarcoplasmic reticulum proteins in failing human dilated cardiomyopathy. Circulation. 1995;92(4):778-784.

59. Schmidt U, Hajjar RJ, Kim CS, Lebeche D, Doye AA, Gwathmey JK. Human heart failure: cAMP stimulation of SR $\mathrm{Ca}(2+)$-ATPase activity and phosphorylation level of phospholamban. Am J Physiol. 1999;277(2 pt 2):H474-H480.

60. Lompre AM, Lambert F, Lakatta EG, Schwartz $\mathrm{K}$. Expression of sarcoplasmic reticulum $\mathrm{Ca}(2+)$ ATPase and calsequestrin genes in rat heart during ontogenic development and aging. Circ Res. 1991; 69(5):1380-1388.

61. Miyamoto MI, et al. Adenoviral gene transfer of SERCA2a improves left-ventricular function in aortic-banded rats in transition to heart failure. Proc Natl Acad Sci U S A. 2000;97(2):793-798.

62. del Monte F, et al. Restoration of contractile function in isolated cardiomyocytes from failing human hearts by gene transfer of SERCA2a. Circulation. 1999;100(23):2308-2311.

63. Kawase Y, et al. Reversal of cardiac dysfunction after long-term expression of SERCA2a by gene transfer in a pre-clinical model of heart failure. J Am Coll Cardiol. 2008;51(11):1112-1119.

64. Sakata S, et al. Restoration of mechanical and energetic function in failing aortic-banded rat hearts by gene transfer of calcium cycling proteins. J Mol Cell Cardiol. 2007;42(4):852-861.

65. SakataS, et al. Transcoronary gene transfer ofSERCA2a increases coronary blood flow and decreases cardiomyocyte size in a type 2 diabetic rat model. Am JPbysiol Heart Circ Physiol. 2007;292(2):H1204-H1207.

66. Lyon AR, et al. SERCA2a gene transfer decreases sarcoplasmic reticulum calcium leak and reduces ventricular arrhythmias in a model of chronic heart failure. Circ Arrhythm Electrophysiol. 2011;4(3):362-372.

67. Cutler MJ, Wan X, Laurita KR, Hajjar RJ, Rosenbaum DS. Targeted SERCA2a gene expression identifies molecular mechanism and therapeutic target for arrhythmogenic cardiac alternans. Circ Arrbythm Electrophysiol. 2009;2(6):686-694.

68. del Monte F, et al. Abrogation of ventricular 
arrhythmias in a model of ischemia and reperfusion by targeting myocardial calcium cycling. Proc Natl Acad Sci U S A. 2004;101(15):5622-5627.

69. Prunier F, et al. Prevention of ventricular arrhythmias with sarcoplasmic reticulum $\mathrm{Ca} 2+$ ATPase pump overexpression in a porcine model of ischemia reperfusion. Circulation. 2008;118(6):614-624.

70. Hadri L, et al. SERCA2a gene transfer enhances eNOS expression and activity in endothelial cells. Mol Ther. 2010;18(7):1284-1292.

71. del Monte F, Harding SE, Dec GW, Gwathmey JK, Hajjar RJ. Targeting phospholamban by gene transfer in human heart failure. Circulation. 2002; 105(8):904-907.

72. Suckau L, et al. Long-term cardiac-targeted RNA interference for the treatment of heart failure restores cardiac function and reduces pathological hypertrophy. Circulation. 2009;119(9):1241-1252.

73. Nicolaou $P$, et al. Inducible expression of active protein phosphatase- 1 inhibitor- 1 enhances basal cardiac function and protects against ischemia/ reperfusion injury. Circ Res. 2009;104(8):1012-1020

74. Pathak A, Baldwin B, Kranias EG. Key protein alterations associated with hyperdynamic cardiac function: insights based on proteomic analysis of the protein phosphatase 1 inhibitor- 1 overexpress- ing hearts. Hellenic J Cardiol. 2007;48(1):30-36.

75. Pathak A, et al. Enhancement of cardiac function and suppression of heart failure progression by inhibition of protein phosphatase 1. Circ Res. 2005; 96(7):756-766.

76. Most P, et al. Cardiac adenoviral S100A1 gene delivery rescues failing myocardium. J Clin Invest. 2004; 114(11):1550-1563.

77. Pleger ST, et al. Stable myocardial-specific AAV6 S100A1 gene therapy results in chronic functional heart failure rescue. Circulation. 2007; 115(19):2506-2515.

78. Most P, Koch WJ. S100A1: a calcium-modulating inotropic prototype for future clinical heart failure therapy. Future Cardiol. 2007;3(1):5-11.

79. Kho C, et al. SUMO1-dependent modulation of SERCA2a in heart failure. Nature. 2011; 477(7366):601-605

80. Ghadge SK, Muhlstedt S, Ozcelik C, Bader M. SDF-1alpha as a therapeutic stem cell homing factor in myocardial infarction. Pharmacol Ther. 2011; 129(1):97-108.

81. Kawase Y, Ladage D, Hajjar RJ. Rescuing the failing heart by targeted gene transfer. J Am Coll Cardiol. 2011;57(10):1169-1180

82. Pyo RT, et al. CXCR4 modulates contractility in adult cardiac myocytes. J Mol Cell Cardiol. 2006; 41(5):834-844

83. Chen J, et al. Effects of CXCR4 gene transfer on cardiac function after ischemia-reperfusion injury. Am J Pathol. 2010;176(4):1705-1715.

84. LaRocca TJ, et al. beta2-Adrenergic receptor signaling in the cardiac myocyte is modulated by interactions with CXCR4. J Cardiovasc Pharmacol. 2010;56(5):548-559.

85. Bainbridge JW, et al. Effect of gene therapy on visual function in Leber's congenital amaurosis. N Engl J Med. 2008;358(21):2231-2239.

86. Hajjar RJ, et al. Design of a phase $1 / 2$ trial of intracoronary administration of AAV1/SERCA2a in patients with heart failure. J Card Fail. 2008; 14(5):355-367.

87. Jaski BE, et al. Calcium upregulation by percutaneous administration of gene therapy in cardiac disease (CUPID Trial), a first-in-human phase $1 / 2$ clinical trial. J Card Fail. 2009;15(3):171-181.

88. Jessup M, et al. Calcium Upregulation by Percutaneous Administration of Gene Therapy in Cardiac Disease (CUPID): a phase 2 trial of intracoronary gene therapy of sarcoplasmic reticulum $\mathrm{Ca} 2+-$ ATPase in patients with advanced heart failure. Circulation. 2011;124(3):304-313. 